FERMILAB-Pub-00/300-T

VAND-TH-00-8

hep-ph/0011247

November 20, 2000

\title{
Optimizing T-violating Effects for Neutrino Oscillations in Matter
}

\author{
Stephen J. Parke ${ }^{1}$ and Thomas J. Weiler ${ }^{2}$ \\ ${ }^{1}$ Theoretical Physics Department, \\ Fermi National Accelerator Laboratory, Batavia, IL 60510, U.S.A. \\ 2 Department of Physics $\& 3$ Astronomy, Vanderbilt University, \\ Nashville, TN 37235, U.S.A.
}

\begin{abstract}
Matter effects may strongly enhance the Jarlskog factor $J$ in T and CP-violating threeneutrino oscillation probabilities. However, we show that when $J$ is enhanced, the same matter effects suppress the oscillating factors and increase the oscillation length. The net result is that there is no large enhancement in measurable probabilities for earthbound experiments using neutrino parameters suggested by current experiments. We show that by an appropriate choice of the experimental parameters, neutrino energy and travel length, the T-violating probability can be enhanced by matter effects over their vacuum values by $50 \%$. Our approach is analytical, allowing considerable insight into the underlying physics.
\end{abstract}

\section{Introduction}

Analogous to the CKM mixing-matrix in the quark sector of the Standard Model (SM), there is an MNS mixing-matrix [1] in the lepton sector. For three light neutrinos (assumed throughout this work) the MNS matrix consists of three angles and one (three) phase(s) for 
Dirac (Majorana) neutrinos. Regardless of the nature of the neutrinos, Dirac vs. Majorana, neutrino oscillation experiments are sensitive to a single phase through the measurement of a T-violating asymmetry $P\left(\nu_{\alpha} \rightarrow \nu_{\beta}\right)-P\left(\nu_{\beta} \rightarrow \nu_{\alpha}\right)$ or a CP-violating asymmetry $P\left(\nu_{\alpha} \rightarrow\right.$ $\left.\nu_{\beta}\right)-P\left(\bar{\nu}_{\alpha} \rightarrow \bar{\nu}_{\beta}\right)$. Much progress has been made toward determining the values of the three mixing angles. From measurements of the neutrino survival probabilities $\nu_{\mu} \rightarrow \nu_{\mu}$ and $\nu_{e} \rightarrow \nu_{e}$ in the atmospheric flux, one infers that one mixing-angle is near maximal $(\pi / 4)$, and one is small [2], the latter statement supported also by the Chooz experiment [3]. From the $\nu_{e} \rightarrow \nu_{e}$ survival probability in the solar flux, one infers that the third angle is either large (for the large-angle MSW (LAM) and the long-wavelength vacuum (LWV) solar solutions), or very small (for the small-angle MSW (SAM) solar solution) [4]. Nothing is known about the $\mathrm{T}$ and $\mathrm{CP}$-violating phase.

With construction underway for long-baseline terrestrial oscillation experiments, attention has turned toward more precise measurements of the MNS parameters, including the phase. Measurement of the $\mathrm{T}$ and $\mathrm{CP}$-violating asymmetries appears impossible for the SAM and LWV solutions. For the SAM solution, this is because of the smallness of two of the three angles, while for the LWV solution, this is because of the extreme hierarchy of mass-squared values $\delta m_{\text {sun }}^{2} / \delta m_{\text {atm }}^{2} \sim 10^{-7} \mathrm{eV}^{2}$. On the other hand, for the LAM solution, measuring $\mathrm{T}$ and $\mathrm{CP}$-violating asymmetries to determine the phase $\delta$ is more promising, with effects at the per cent level. In terrestrial experiments, the neutrino beam will travel underground, and one may ask whether earth-matter effects [5] can enhance the $\mathrm{T}$ and $\mathrm{CP}$-violating probabilities [6].

Throughout this paper, we assume a three neutrino world, and the vacuum hierarchy $\delta m_{21}^{2} \ll \delta m_{32}^{2}$ as indicated by experiment. We focus on T-violation, defined by the asymmetry

$$
P^{\mathrm{T}} \equiv P\left(\nu_{\alpha} \rightarrow \nu_{\beta}\right)-P\left(\nu_{\beta} \rightarrow \nu_{\alpha}\right)
$$

Here $\alpha$ and $\beta$ denote different neutrino (or anti-neutrino) flavors. The CP-violating asymmetry, defined by

$$
P^{\ell म P} \equiv P\left(\nu_{\alpha} \rightarrow \nu_{\beta}\right)-P\left(\bar{\nu}_{\alpha} \rightarrow \bar{\nu}_{\beta}\right)
$$

contains the same contribution as $P^{T}$ but includes additional contributions arising solely from matter effects. We do not pursue this complication in this work. Here we analyze the case for 
a measurable T-violation. Time-reversing the path of the neutrino through the earth gives no extrinsic T-violation (for a spherically symmetric earth-matter distribution). This fact, and the relatively small effects of matter on $P^{T}$ derived herein, make the T-violation measurement an attractive approach for extracting the intrinsic T and CP-violating parameter $\delta$.

For certain small values of mixing angles and for certain neutrino energies, strong enhancements may occur for the Jarlskog invariant $J$ in matter (m) relative to vacuum (v) [7]. It is not hard to see why $J_{\mathrm{m}}$ can be strongly enhanced near a small-angle MSW resonance. In the two flavor approximation, which is valid for three neutrinos with a hierarchy of vacuum mass-squared differences $\delta m_{21}^{2} \ll \delta m_{32}^{2}$, the relation $\delta m_{\mathrm{m}}^{2}=\sin \left(2 \theta_{\mathrm{v}}\right) \delta m_{\mathrm{v}}^{2}$ obtained at resonance, implies $J_{\mathrm{m}} \propto 1 / \theta_{\mathrm{v}}$ for small mixing-angle. The purpose of this Letter is to show that although $J_{\mathrm{m}}$ may be strongly enhanced by small-angle matter resonances, the measurable T-violating neutrino oscillation probability proportional to $J_{\mathrm{m}}$ is not enhanced for earth-bound experiments. In fact, depending on the exact values of the mixing angles and vacuum masses, matter effects, if important at all, generally suppress these probabilities. However, we do show that for an appropriate choice of neutrino energy and travel distance, matter effects give modest $(\sim 50 \%)$ enhancements of the T-violating probabilities for terrestrial experiments. Our results are mainly analytical, and so some insight into the physics of the matter phenomenon emerges.

\section{Enhancement of T-Violating Probabilities?}

The reason for the absence of a significant enhancement in probability is easy to understand, as we now demonstrate. For oscillations in vacuum the T-violating probability, eq. (1), is simply

$$
P_{\mathrm{v}}^{\mathrm{T}}=16 J_{\mathrm{v}} \sin \Delta_{21}^{\mathrm{v}} \sin \Delta_{32}^{\mathrm{v}} \sin \Delta_{31}^{\mathrm{v}}
$$

where

$$
\Delta_{j k}^{\mathrm{v}}=\frac{\left.\delta m_{j k}^{2}\right|_{\mathrm{v}} L}{4 E_{\nu}}=1.2669 \cdots \frac{\left(L / 10^{3} \mathrm{~km}\right)\left(\delta m_{j k}^{2} / 10^{-3} \mathrm{eV}^{2}\right)}{(E / \mathrm{GeV})} ;
$$

$\left.\delta m_{j k}^{2}\right|_{\mathrm{v}}$ is the difference of $j^{\text {th }}$ and $k^{\text {th }}$ vacuum mass-squared eigenvalues, $E_{\nu}$ is the neutrino energy, and $L$ is the travel distance. The Jarlskog factor [8], $J$, in the standard mixing 
parameterization [9] is given by

$$
J_{\mathrm{v}}=\left[s_{21} s_{31} s_{32} c_{21} c_{31}^{2} c_{32} \sin \delta\right]_{\mathrm{v}}
$$

where $s_{21} \equiv \sin \theta_{21}$, etc. $J_{\mathrm{v}}$ has a maximum value of $\frac{1}{6 \sqrt{3}}$. Whereas for oscillations in matter of constant densityĐ we have

$$
P_{\mathrm{m}}^{\mathrm{T}}=16 J_{\mathrm{m}} \sin \Delta_{21}^{\mathrm{m}} \sin \Delta_{32}^{\mathrm{m}} \sin \Delta_{31}^{\mathrm{m}},
$$

where the $m$ sub- or superscript indicates the value in matter. Again the maximum value of $J_{m}=\left[s_{21} s_{31} s_{32} c_{21} c_{31}^{2} c_{32} \sin \delta\right]_{\mathrm{m}}$ is $\frac{1}{6 \sqrt{3}}$. If we now employ the elegant three generation relation [10

$$
\frac{J_{\mathrm{m}}}{J_{\mathrm{v}}}=\frac{\left[\delta m_{21}^{2} \delta m_{32}^{2} \delta m_{31}^{2}\right]_{\mathrm{v}}}{\left[\delta m_{21}^{2} \delta m_{32}^{2} \delta m_{31}^{2}\right]_{\mathrm{m}}}
$$

relating vacuum and matter Jarlskog factors and mass-squared differences, then the Tviolation asymmetry in matter can be written as

$$
P_{\mathrm{m}}^{\mathrm{T}}=16 J_{\mathrm{v}}\left[\frac{\left.\left(\delta m_{21}^{2} \delta m_{32}^{2} \delta m_{31}^{2}\right)\right|_{\mathrm{v}}}{\left.\left(\delta m_{21}^{2} \delta m_{32}^{2} \delta m_{31}^{2}\right)\right|_{\mathrm{m}}}\right] \sin \Delta_{21}^{\mathrm{m}} \sin \Delta_{32}^{\mathrm{m}} \sin \Delta_{31}^{\mathrm{m}} .
$$

For small distances such that $\sin \Delta \approx \Delta$ for all $\Delta$ 's, both in vacuum and matter, then $P_{\mathrm{v}}^{\mathrm{T}} \approx P_{\mathrm{m}}^{\mathrm{T}}$. That is, at these distances matter effects are negligible. At longer lengths, the bracketed ratio in eq.(8) can provide an enhancement if one of the $\left.\delta m^{2}\right|_{\mathrm{m}}$ becomes small compared to its vacuum value, i.e. near a resonance. The first resonance is encountered when

$$
E_{\nu} \simeq \frac{\left.\delta m_{21}^{2}\right|_{\mathrm{v}} \cos 2 \theta_{21}^{\mathrm{v}}}{2 \sqrt{2} G_{F} N_{e}} \equiv E_{\nu}^{1 R}
$$

for which

$$
\left.\left.\delta m_{21}^{2}\right|_{\mathrm{m}} \simeq \delta m_{21}^{2}\right|_{\mathrm{v}} \sin 2 \theta_{21}^{\mathrm{v}} \quad \text { and }\left.\left.\quad \delta m_{32}^{2}\right|_{\mathrm{m}} \simeq \delta m_{32}^{2}\right|_{\mathrm{v}}
$$

and the bracketed expression in eq. (8) becomes

$$
\frac{1}{\sin 2 \theta_{21}^{\mathrm{v}}}
$$

The second resonance occurs when

$$
E_{\nu} \simeq \frac{\left.\delta m_{32}^{2}\right|_{\mathrm{v}} \cos 2 \theta_{31}^{\mathrm{v}}}{2 \sqrt{2} G_{F} N_{e}} \equiv E_{\nu}^{2 R},
$$

\footnotetext{
${ }^{1}$ In the earth this approximation is quite accurate for those paths that do not enter the earth's core; for paths that do enter the core, the constant density approximation gives qualitatively the correct physics.
} 
for which

$$
\left.\left.\delta m_{32}^{2}\right|_{\mathrm{m}} \simeq \delta m_{32}^{2}\right|_{\mathrm{v}} \sin 2 \theta_{31}^{\mathrm{v}} \quad \text { and }\left.\left.\quad \delta m_{21}^{2}\right|_{\mathrm{m}} \simeq \delta m_{32}^{2}\right|_{\mathrm{v}}
$$

and the bracketed expression in eq. (8) becomes

$$
\frac{\left.\delta m_{21}^{2}\right|_{\mathrm{v}}}{\left.\delta m_{32}^{2}\right|_{\mathrm{v}} \sin 2 \theta_{31}^{\mathrm{v}}} .
$$

To illustrate how matter affects the Jarlskog factor, $J_{\mathrm{m}}$, we have chosen representative values for both the earth's density and the neutrino vacuum parameters: a matter density typical of the earth's mantle $\left(\sim 3 \mathrm{~g} \mathrm{~cm}^{-3}\right)$ and neutrino masses and mixing which are centered in the allowed regions for the atmospheric neutrinos and the large angle MSW solar solution. The chosen vacuum values are

$$
\begin{array}{cc}
\left.\delta m_{32}^{2}\right|_{\mathrm{v}}=3.5 \times 10^{-3} \mathrm{eV}^{2} & \sin ^{2} 2 \theta_{32}^{\mathrm{v}}=1 \\
\left.\delta m_{21}^{2}\right|_{\mathrm{v}}=5.0 \times 10^{-5} \mathrm{eV}^{2} \quad \sin ^{2} 2 \theta_{21}^{\mathrm{v}}=0.8 \\
\left.\delta m_{31}^{2}\right|_{\mathrm{v}}=3.5 \times 10^{-3} \mathrm{eV}^{2} \quad \sin ^{2} 2 \theta_{31}^{\mathrm{v}}=0.03 \\
\sin \delta^{\mathrm{v}}=1 \\
Y_{e} \rho=1.5 \mathrm{~g} \mathrm{~cm}^{-3} .
\end{array}
$$

The CP and T-violating angle $\delta^{\mathrm{v}}$ has been chosen to be maximal, $\frac{\pi}{2}$. The value of $\sin ^{2} 2 \theta_{31}^{\mathrm{v}}$ is chosen to be half an order of magnitude below the Chooz limit [3]. Using these parameter values, the matter mass-eigenvalues and the ratio $J_{\mathrm{m}} / J_{\mathrm{v}}$, equal to the bracketed ratio of eq. (8), is given in fig. 11. Note that away from the resonances, $J_{\mathrm{m}} / J_{\mathrm{v}}$ is much less than one, suggesting that matter effects suppress the amplitude of the T-violating oscillation. In contrast, there are peaks in the ratio $J_{\mathrm{m}} / J_{\mathrm{v}}$ near the resonances.

In principle, the peaks in $J_{\mathrm{m}} / J_{\mathrm{v}}$ at the resonances become significant enhancements, $\gg 1$, when either

$$
\sin 2 \theta_{21}^{\mathrm{v}} \ll 1, \quad \text { or } \quad \sin 2 \theta_{31}^{\mathrm{v}} \ll \frac{\left.\delta m_{21}^{2}\right|_{\mathrm{v}}}{\left.\delta m_{32}^{2}\right|_{\mathrm{v}}} \lesssim \mathcal{O}\left(10^{-2}\right),
$$

holds. (Neither of these conditions is satisfied in our representative example.) However if either of these conditions holds, then the vacuum Jarlskog factor $J_{\mathrm{v}}$ of eq. (5) is itself very small. The message here is that the Jarlskog factor can be strongly enhanced by matter, as in [7], only when the vacuum value is very small to begin with; the enhancement never 

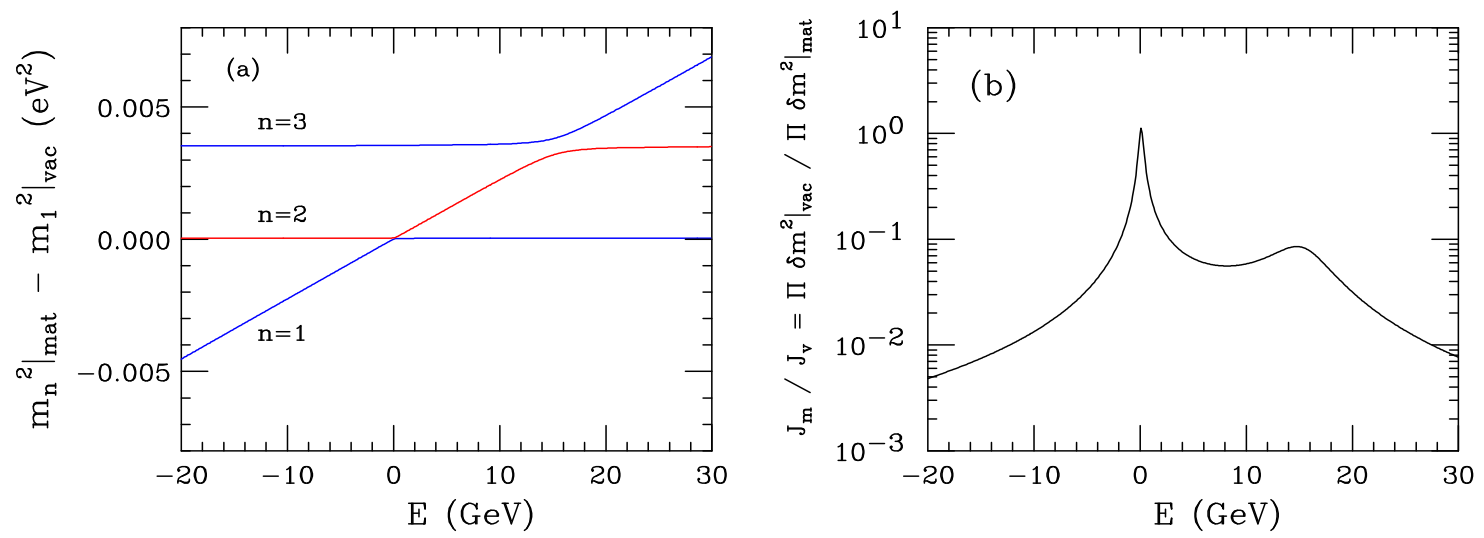

Figure 1: Shown are (a) the neutrino mass-squared eigenvalues in matter and (b) the ratio $J_{\mathrm{m}} / J_{\mathrm{v}}$, for the parameters listed in eq. (15), as a function of the neutrino energy. Positive energies correspond to neutrinos, and negative energies correspond to anti-neutrinos (vice versa for inverted $\delta m^{2}$ 's).

produces a large value of $J_{\mathrm{m}}$. A quantitative view of the impossibility of matter to produce a truly large amplitude results when the explicit expression for $J_{\mathrm{v}}$ in eq. (5) is substituted into eq. (8). The result is

$P_{\mathrm{m}}^{\mathrm{T}}=2 \cos \theta_{31}^{\mathrm{v}} \sin \left(\delta^{\mathrm{v}}\right)\left[\frac{\left[\left(\sin 2 \theta_{21} \delta m_{21}^{2}\right)\left(\sin 2 \theta_{32} \delta m_{32}^{2}\right)\left(\sin 2 \theta_{31} \delta m_{31}^{2}\right)\right]_{\mathrm{v}}}{\left[\begin{array}{lll}\delta m_{21}^{2} & \delta m_{32}^{2} & \delta m_{31}^{2}\end{array}\right]_{\mathrm{m}}}\right] \sin \Delta_{21}^{\mathrm{m}} \sin \Delta_{32}^{\mathrm{m}} \sin \Delta_{31}^{\mathrm{m}}$.

As seen from eqs. (10) and (13), at either resonance the bracketed factor in this equation does not become large. What the resonance manages to do is to cancel the small vacuum value of $\sin 2 \theta_{21}^{\mathrm{v}}$ or $\sin 2 \theta_{31}^{\mathrm{v}}$ in the amplitude $\left(16 J_{\mathrm{v}}\right)$ of the T-violating oscillation. But accompanying even this cancellation is a negative consequence for the associated oscillation lengths, to which we now turn.

\section{Baseline Limitations}

A significant enhancement of T-violating oscillation amplitudes requires a small-angle resonance. The conditions for this are either

$$
\left.\left.\delta m_{21}^{2}\right|_{\mathrm{m}} \ll \delta m_{21}^{2}\right|_{\mathrm{v}} \quad \text { or }\left.\left.\quad \delta m_{32}^{2}\right|_{\mathrm{m}} \ll \delta m_{21}^{2}\right|_{\mathrm{v}} .
$$


These conditions in turn imply that the oscillation length in matter associated with the smallest $\delta m^{2}$ is larger than the largest oscillation length in vacuum, the one associated with the smallest vacuum $\delta m^{2}$, i.e. $\left.\delta m_{21}^{2}\right|_{\mathrm{v}}$. Thus, there is the danger that for an enhanced amplitude, the baseline requirement will exceed the capability of a terrestrial experiment. In fact, this happens.

Assuming hierarchical mass splittings, the fast oscillation governed by the larger

$$
\Delta_{+}^{\mathrm{m}} \equiv \max \left[\Delta_{21}^{\mathrm{m}}, \Delta_{32}^{\mathrm{m}}\right] \equiv \frac{\delta m_{+}^{2} L}{4 E_{\nu}}
$$

will be amplitude modulated by the slow oscillation, governed by

$$
\Delta_{-}^{\mathrm{m}} \equiv \min \left[\Delta_{21}^{\mathrm{m}}, \Delta_{32}^{\mathrm{m}}\right] \equiv \frac{\delta m_{-}^{2} L}{4 E_{\nu}}
$$

The consequence is that the T-violating oscillation is bounded above by $\sin \left(\Delta_{-}^{\mathrm{m}}\right) \cos ^{2}\left(\Delta_{-}^{\mathrm{m}} / 2\right)$, and below by $-\sin \left(\Delta_{-}^{\mathrm{m}}\right) \sin ^{2}\left(\Delta_{-}^{\mathrm{m}} / 2\right)$. An idealized experiment would resolve the fast oscillation and measure the difference of the bounding envelopes. This difference is just $\sin \left(\Delta_{-}^{\mathrm{m}}\right)$, which is maximized at $(2 n+1) \pi / 2$. However, a realistic measurement will average over the fast oscillation, thereby measuring the mean of the envelopes given by $\frac{1}{4} \sin \left(2 \Delta_{-}^{\mathrm{m}}\right)$, which is maximized at $\Delta_{-}^{\mathrm{m}}=(2 n+1) \frac{\pi}{4}$. The choice $n=0$ minimizes the source-detector distance, and therefore optimizes the incident flux. So we are led to consider $\Delta_{-}^{\mathrm{m}}=\frac{\pi}{4}$ as the condition which maximizes the size of the T-violating asymmetry. The corresponding distance, $L_{\mathrm{m}}^{\mathrm{T}}$, is given by

$$
L_{\mathrm{m}}^{\mathrm{T}}=\frac{\pi E_{\nu}}{\delta m_{-}^{2}}
$$

which at the first or second resonance (eqs. (9) and (10), or (12) and (13)) becomes

$$
L_{\mathrm{m}}^{\mathrm{T}}=\frac{0.2 D_{\oplus}}{\tan 2 \theta_{21}^{\mathrm{v}}} \quad \text { or } \quad \frac{0.2 D_{\oplus}}{\tan 2 \theta_{31}^{\mathrm{v}}}
$$

when the conversion

$$
\frac{\pi}{2 \sqrt{2} G_{F} N_{e}}=0.2 D_{\oplus}
$$

is used to relate the matter scale to the earth's diameter, $D_{\oplus} \sim 13,000 \mathrm{~km}$. This latter conversion holds for the mantle density given in eq.(16) - the matter density is a factor of 2 larger (smaller) in the earth's core (outer crust). For a significant amplitude enhancement to occur the corresponding $\theta^{\mathrm{v}}$ is necessarily small. Therefore it follows that when $J_{\mathrm{m}} \gg J_{\mathrm{v}}$, 
then $L_{\mathrm{m}}^{\mathrm{T}}$ approaches or exceeds the diameter of the earth. This in turn ensures that for terrestrial experiments with $L \ll L_{\mathrm{m}}^{\mathrm{T}}$, the resonant $\delta m_{-}^{2}$ is such that $\sin \Delta_{-}^{\mathrm{m}} \sim \Delta_{-}^{\mathrm{m}} \ll 1$ and that $P_{\mathrm{m}}^{\mathrm{T}}$ is of the same order as $P_{\mathrm{v}}^{\mathrm{T}}$, i.e. no large enhancement.

\section{Magnitude of the First Peaks}

Two arguments may be made for the importance of the very first, or first few, peaks as the target for measurement. The first argument is that the T-violating oscillation length in the earth, as discussed above, is characteristically of order of the earth's diameter or longer. Thus, even long baseline experiments may be limited to the first few peaks. The second argument is that the $1 / L^{2}$ fall-off of the incident neutrino flux for any aperture-limited experiment favors shorter distances. $]^{2}$

In vacuum, the first few peaks in the T-violating asymmetry given by eq. (3) occur when

$\sin \Delta_{21}^{\mathrm{v}} \sin \Delta_{32}^{\mathrm{v}} \sin \Delta_{31}^{\mathrm{v}} \approx \frac{\left.\delta m_{21}^{2}\right|_{\mathrm{v}}}{\left.\delta m_{32}^{2}\right|_{\mathrm{v}}} \Delta_{32}^{\mathrm{v}} \sin ^{2} \Delta_{32}^{\mathrm{v}}$ is maximized. The rough location of these peaks is given by

$$
\frac{L_{\mathrm{v}}}{E_{\nu}} \sim(2 n+1) 2 \pi \frac{1}{\left.\delta m_{32}^{2}\right|_{\mathrm{v}}},
$$

for $\mathrm{n}=0,1,2 \cdots$ until the approximation $\sin \Delta_{21}^{\mathrm{v}} \approx \Delta_{21}^{\mathrm{v}}$ is no longer valid. The asymmetry at the $n$-th peak is equal to

$$
(2 n+1) 8 \pi J_{\mathrm{v}} \frac{\left.\delta m_{21}^{2}\right|_{\mathrm{v}}}{\left.\delta m_{32}^{2}\right|_{\mathrm{v}}} .
$$

Thus the asymmetry and the distance to the $n$-th peak both grow as $(2 n+1)$. In particular the second peak is three times larger than the first peak and three times further out. Remember however that the neutrino beam intensity falls as $L^{-2}$ for fixed $E_{\nu}$, disfavoring the more distant peaks.

For the first peak, a numerical calculation produces an estimate more accurate than eqs. (24) and (25); the result is

$$
\Delta_{32}^{\mathrm{v}} \sim \frac{7 \pi}{12}, \quad \text { i.e. } \quad \frac{L_{\mathrm{v}}}{E_{\nu}} \sim \frac{7 \pi}{3} \frac{1}{\left.\delta m_{32}^{2}\right|_{\mathrm{v}}},
$$

\footnotetext{
${ }^{2}$ When $\sin \left(\Delta_{-}^{\mathrm{m}}\right) \sim \Delta_{-}^{\mathrm{m}}$ applies, one power of $L^{-1}$ is compensated.
} 
with the size of the asymmetry at the first peak being

$$
\sim 8.7 \pi J_{\mathrm{v}} \frac{\left.\delta m_{21}^{2}\right|_{\mathrm{v}}}{\left.\delta m_{32}^{2}\right|_{\mathrm{v}}} .
$$

So even the first peak is reasonably approximated by the general results above.

In matter, the physics is more complex since the $\delta m^{2}$ 's change with energy. We start by looking at the product of the three sine terms

$$
\sin \left(\Delta_{-}^{\mathrm{m}}\right) \sin \left(\Delta_{+}^{\mathrm{m}}\right) \sin \left(\Delta_{-}^{\mathrm{m}}+\Delta_{+}^{\mathrm{m}}\right)
$$

The first peak of this product occurs when

$$
\Delta_{-}^{\mathrm{m}}+\Delta_{+}^{\mathrm{m}} \sim \frac{7 \pi}{12} \quad \text { if } \quad \Delta_{-}^{\mathrm{m}} \ll \Delta_{+}^{\mathrm{m}},
$$

and at

$$
\Delta_{-}^{\mathrm{m}}+\Delta_{+}^{\mathrm{m}}=\frac{2 \pi}{3} \quad \text { if } \quad \Delta_{-}^{\mathrm{m}}=\Delta_{+}^{\mathrm{m}} .
$$

In terms of $\left(\Delta_{-}^{\mathrm{m}}+\Delta_{+}^{\mathrm{m}}\right)$, the first peak moves monotonically from $\sim \frac{7 \pi}{12}$ to $\frac{2 \pi}{3}$ as the ratio of $\Delta_{-}^{\mathrm{m}}$ to $\Delta_{+}^{\mathrm{m}}$ changes from 0 to 1 . At the first peak this product of the sines may be written as

$$
\eta \frac{2 \Delta_{-}^{\mathrm{m}} \Delta_{+}^{\mathrm{m}}}{\left(\Delta_{-}^{\mathrm{m}}+\Delta_{+}^{\mathrm{m}}\right)^{2}}=\eta \frac{2 \delta m_{-}^{2} \delta m_{+}^{2}}{\left(\delta m_{-}^{2}+\delta m_{+}^{2}\right)^{2}}
$$

where $\eta$ is slowly varying, monotonically increasing, function of $\frac{\Delta_{-}^{\mathrm{m}}}{\Delta_{+}^{\mathrm{m}}}=\frac{\delta m_{-}^{2}}{\delta m_{+}^{2}}$ with $\eta(0) \approx 0.86$ and $\eta(1)=3 \sqrt{3} / 4 \approx 1.30$.

Thus the full T-violating asymmetry, eq.(8), at the first peak is

$$
P_{\mathrm{m}}^{\mathrm{T}}=32 J_{\mathrm{v}} \eta\left[\frac{\left.\left(\delta m_{21}^{2} \delta m_{32}^{2} \delta m_{31}^{2}\right)\right|_{\mathrm{v}}}{\left(\delta m_{-}^{2}+\delta m_{+}^{2}\right)^{3}}\right] .
$$

For energies between the two resonances, eqs.(9) and (12), the following sum-rule holds

$$
\left.\left(\delta m_{-}^{2}+\delta m_{+}^{2}\right) \approx \delta m_{32}^{2}\right|_{\mathrm{v}},
$$

as evidenced in Fig. 1 (a). This is a good approximation provided both $\left.\delta m_{21}^{2}\right|_{\mathrm{v}} \sin 2 \theta_{21}^{\mathrm{v}}$ and $\left.\delta m_{32}^{2}\right|_{\mathrm{v}} \sin 2 \theta_{31}^{\mathrm{v}}$ are much smaller than $\left.\delta m_{32}^{2}\right|_{\mathrm{v}}$.

Below the first resonance and above the second resonance, $\left(\delta m_{-}^{2}+\delta m_{+}^{2}\right)$ grows approximately linearly with energy from the minimum value, $\left.\delta m_{32}^{2}\right|_{\mathrm{v}}$. Since the function $\eta$ varies 
little it cannot compensate for the increase in size of $\left(\delta m_{-}^{2}+\delta m_{+}^{2}\right)$ for energies below the first resonance or above the second resonance. Therefore the maximum value of the first peak of the T-violating asymmetry occurs between the resonances where $\eta$ is maximized, i.e.

$$
\delta m_{-}^{2}=\left.\delta m_{+}^{2} \approx \frac{1}{2} \delta m_{32}^{2}\right|_{\mathrm{v}}
$$

This is shown in Fig. 目(a) where the ratio of the asymmetry in matter and in vacuum at their respective first peaks, is plotted versus energy. (The vacuum value of the first peak is energy independent, eq.(27)).

The neutrino energy for the maximum first peak in matter is given by (recall eq. (12))

$$
\begin{aligned}
E_{\nu} \simeq \frac{1}{2} E_{\nu}^{2 R} & =\frac{\left.\delta m_{32}^{2}\right|_{\mathrm{v}} \cos 2 \theta_{31}^{\mathrm{v}}}{4 \sqrt{2} G_{F} N_{e}} \\
& \sim 7.6 \cos 2 \theta_{31}^{\mathrm{v}}\left[\frac{\left.\delta m_{32}^{2}\right|_{\mathrm{v}}}{3.5 \times 10^{-3} \mathrm{eV}^{2}}\right]\left[\frac{1.5 \mathrm{~g} \mathrm{~cm}^{-3}}{Y_{e} \rho}\right] \mathrm{GeV},
\end{aligned}
$$

and the peak occurs at a distance fixed by $\Delta_{+}^{\mathrm{m}}=\Delta_{-}^{\mathrm{m}}=\pi / 3$, i.e.

$$
L=\frac{8 \pi}{3} \frac{E_{\nu}}{\left.\delta m_{32}^{2}\right|_{\mathrm{v}}}=\frac{4 \pi}{3} \frac{\cos 2 \theta_{31}^{\mathrm{v}}}{2 \sqrt{2} G_{F} N_{e}} \sim 3600 \cos 2 \theta_{31}^{\mathrm{v}}\left[\frac{1.5 \mathrm{~g} \mathrm{~cm}^{-3}}{Y_{e} \rho}\right] \mathrm{km} .
$$

Note that this distance is independent of $\left.\delta m^{2}\right|_{\mathrm{v}}$. The value of the T-violating asymmetry at this peak is

$$
24 \sqrt{3} J_{\mathrm{v}} \frac{\left.\delta m_{21}^{2}\right|_{\mathrm{v}}}{\left.\delta m_{32}^{2}\right|_{\mathrm{v}}} .
$$

The matter asymmetry at this peak is $52 \%$ larger than the asymmetry at the first peak in vacuum, given in eq. (27). From Fig. 1 (b) we see that, for our chosen parameters, matter effects at $E_{\nu}=7.6 \mathrm{GeV}$ actually suppress $J$ by more than an order of magnitude which is more than compensated by an increase in the product of sines due to matter. But at this energy the $L$ value for the first peak in matter exceeds the same in vacuum by $14 \%$.

In Fig. 2(b) we show the repeating (in $L$ ) peaks for the T-violating asymmetry with the energy fixed at one half the second resonant value for both neutrinos and anti-neutrinos in matter and vacuum. For neutrinos this energy gives the largest first peak in matter and all subsequent peaks have the same magnitude but alternate in sign.

To go beyond the first peak in the asymmetry is quite complicated. For the second peak, the general features are that matter effects, if important, suppress the magnitude of the 

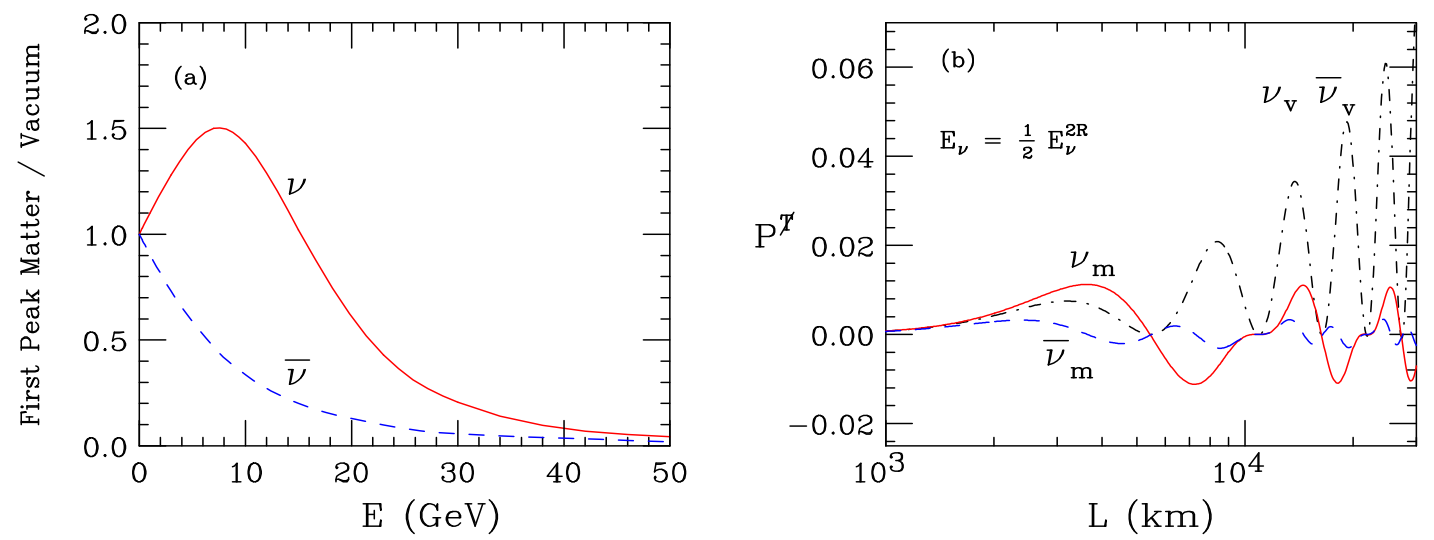

Figure 2: Using the parameters given in eq.(15); Shown are (a) the ratio of the value of the asymmetry at the first peak in matter over vacuum as function of the neutrino energy - for neutrinos the ratio peaks at half the second resonant energy. (b) the asymmetry $P^{\bar{T}}$ versus distance for neutrinos and anti-neutrinos in matter and in vacuum for an energy $(7.6 \mathrm{GeV})$ equal to half of the second resonant value, as specified in eq. (36).

asymmetry compared to vacuum values; also, these effects can flip the sign of the asymmetry compared to the first peak. There are two situations which allow a simple complete analysis. The first situation results when the neutrino energy is chosen so there is a substantial mass hierarchy (i.e. near one of the resonant energies). The size and position of the peaks in this situation closely follow the vacuum case discussed earlier, see eqs. (2427). For the neutrino parameters used in this paper, this situation occurs only at low neutrino energy $<3 \mathrm{GeV}$ or if $\theta_{31}^{\mathrm{v}}$ is small near $E_{\nu}=E_{\nu}^{2 R} \sim 15 \mathrm{GeV}$. However, at the higher-energy resonance, only that part of the beam which is within a few $\mathrm{GeV}$ of the resonance energy, so as to maintain the extreme $\left.\delta m^{2}\right|_{\mathrm{m}}$ hierarchy, contributes to an unsuppressed asymmetry. The second situation results when the neutrino energy and travel length are such that the product of the three sines is at its maximize value. This case is solved in generality in the Appendix and is relevant for case when $E_{\nu}=\frac{1}{2} E_{\nu}^{2 R} \sim 7.6 \mathrm{GeV}$ discussed above.

\section{Summary and Conclusions}

Even though matter effects can significantly enhance the Jarlskog factor in cases where the vacuum value is small, this enhancement does not lead to large enhancements of the T- 
violating probabilities for terrestrial experiments. The reason for this is that associated with this enhancement of the Jarlskog factor is an increase in the longest oscillation length so that for the neutrino parameters suggested by current experiments with small vacuum Jarlskog factor, the enhancement occurs for distances beyond the earth's diameter.

However we have shown that the first peak in the T-violating probability can be enhanced in matter as a result of an enhancement of the oscillating factors, which more than compensates for the suppression of the Jarlskog factor. This first peak is experimentally the most accessible. The enhancement of the first peak occurs for neutrino energies between the two resonant energies and has a broad maximum midway between these two resonant energies that is $50 \%$ larger than the first peak in vacuum. The enhanced first peak occurs

for a neutrino travel distance of $2 \pi \cos 2 \theta_{31}^{\mathrm{v}} / 3 \sqrt{2} G_{F} N_{e}$ which is $\sim 3600 \mathrm{~km}$. Note that this distance depends only on the density of the earth and not on the parameters of the neutrinos (assuming $\cos 2 \theta_{31}^{\mathrm{v}} \sim 1$ ).

Away from the resonant energies the second and higher peaks in the T-violating probability are generally suppressed compared to their growing vacuum counter parts. For neutrinos with energies higher than the higher resonant energy and for anti-neutrinos of all energies the first peak in matter is suppressed compared to the corresponding peak in vacuum. Thus the optimum selectable parameters for the observation of T-violation are a neutrino energy midway between the resonance energies and a travel length of $\sim 3600 \mathrm{~km}$.

Application of these ideas to the experimentally more accessible CP-violation using neutrino factory beams is under investigation.

\section{Acknowledgements}

We thank the Aspen Center for Physics for providing a stimulating working environment were this work began. SP would like to thank Boris Kayser for useful discussions. This work was supported in part by the DOE grant no. DE-FG05-85ER40226, and Fermilab is operated by URA under DOE contract No. DE-AC02-76CH03000. 


\section{References}

[1] Z. Maki, M. Nakagawa, and S. Sakata, Prog. Theo. Phys. 28, 870 (1962).

[2] Reviewed by H. Sobel (SuperK Collaboration), at Neutrino 2000, Sudbury, Canada, June 2000.

[3] CHOOZ Collaboration, M. Apollonio et al., Phys. Lett. B 420, 397 (1998).

[4] Reviewed by Y. Suzuki (SuperK Collaboration), at Neutrino 2000, Sudbury, Canada, June 2000.

[5] L. Wolfenstein, Phys. Rev. D 17, 2369 (1978); V. Barger, S. Pakavasa, R.J.N. Phillips, and K. Whisnant, Phys. Rev. D 22, 2718 (1980); T.K. Kuo and J. Pantaleone, Rev. Mod. Phys. 61, 937 (1989); H.W. Zaglauer and K.H. Schwarzer, Z. Phys. C 40, 273 (1988); P. Krastev, Nuovo Cim. A103, 361 (1990); R.H. Bernstein and S.J. Parke, Phys. Rev. D 44, 2069 (1991).

[6] Recently many authors have revised aspects of matter-effects on three-neutrino oscillations in proposed long and medium baseline experiments. See, e.g., J. Arafune, M. Koike, and J. Sato, Phys. Rev. D56, 3093 (1997); S.M. Bilenky, C. Giunti, W.Grimus, Phys. Rev. D58, 033001 (1998); S. Geer, Phys. Rev. D 57, 6989 (1998); M. Tanimoto, Phys. Lett. B462, 115 (1999); A. De Rujula, M.B. Gavela, and P. Hernández, Nucl. Phys. B 547, 21 (1999); K. Dick, M. Freund, M. Lindner, and A. Romanino, Nucl. Phys. B 562, 299 (1999); V. Barger, S. Geer, and K. Whisnant, Phys. Rev. D 61, 053004 (2000); V. Barger, S. Geer, R. Raja, and K. Whisnant, Phys. Rev. D 62, 013004 (2000); A. Donini, M.B. Gavela, P. Hernández, and S. Rigolin, Nucl. Phys. B 574, 23 (2000); A. Cervera et al., Nucl. Phys. B579, 17 (2000); P. Lipari, Phys. Rev. D 61, 113004 (2000); O. Yasuda, Acta. Phys. Polon. B 30, 3089 (1999); M. Freund, M. Lindner, S.T. Petcov, A. Romanino, Nucl. Phys. B 578, 27 (2000); M. Koike and J. Sato, Phys. Rev. D61, 073012 (2000); M. Freund, T. Ohlsson, Mod. Phys. Lett. A15, 867 (2000); T. Ohlsson and H. Snellman, Phys. Lett. B474, 153 (2000); M. Campanelli, A. Bueno, A. Rubbia, hep-ph/9905240 I. Mocioiu and R. Shrock, hep-ph/0002149, Phys. Rev. D62, 
053017 (2000); H. Minikata and H. Nunokawa, hep-ph/0004114 and hep-ph/0009091; B. Richter, hep-ph/000822; Z.Z. Xing, in [7].

[7] P. Krastev and S. Petcov, Phys. Lett. B205, 84 (1988); M. Koike and J. Sato, Phys. Rev. D62, 073006 (2000); H. Yokomakura, K. Kimura, and A. Takamura, hep-ph/0009141; Z.Z. Xing, hep-ph/0009294.

[8] C. Jarlskog, Phys. Rev. Lett. 55, 1039 (1985).

[9] Particle Data Group, Euro. Phys. J., 15, p. 110 (2000).

[10] P.F. Harrison and W.G. Scott, Phys. Lett. B 476, 349 (2000)

\section{APPENDIX: Solutions for Maximum Peaks}

In this appendix we derive the energy, distance, and peak height associated with each maximum product $\sin \left(\Delta_{-}^{\mathrm{m}}\right) \sin \left(\Delta_{+}^{\mathrm{m}}\right) \sin \left(\Delta_{+}^{\mathrm{m}}+\Delta_{-}^{\mathrm{m}}\right)$. Above $\sim 2 \mathrm{GeV}$, safely away from the lowestenergy resonance, the prefactor $J_{\mathrm{m}} / J_{\mathrm{v}}$, exhibited in Fig. 1b, is a relatively slowly-varying function of $E$. Thus, we expect the conditions for the peaks in the asymmetry to be wellapproximated by the conditions for the maxima in the product of sines. The error inherent in this approximation is almost certainly less than the observational error in the neutrino energy inferred from the charged-current measurement.

The largest value of the product $\sin \left(\Delta_{-}^{\mathrm{m}}\right) \sin \left(\Delta_{+}^{\mathrm{m}}\right) \sin \left(\Delta_{-}^{\mathrm{m}}+\Delta_{+}^{\mathrm{m}}\right)$ is $(\sqrt{3} / 2)^{3}$ when the energy of the neutrinos and the detector distance $L$ are such that two conditions on the phases of the slow and fast oscillations are met. The conditions are

$$
\Delta_{ \pm}=\frac{\pi}{3} n_{ \pm}
$$

where

$$
n_{-} \text {and } n_{+}=1(\bmod 3) \quad \text { or } 2(\bmod 3) \text {, }
$$

and $n_{+} \geq n_{-}$by definition. One of the conditions in eq. (39) may be replaced by an equivalent commensurability condition,

$$
\frac{\Delta_{-}^{\mathrm{m}}}{\Delta_{+}^{\mathrm{m}}}=\frac{\delta m_{-}^{2}}{\delta m_{+}^{2}}=\frac{n_{-}}{n_{+}}
$$


With two variables $(E$ and $L)$ under experimental control, the two independent conditions of eqs. (39 41) can always be satisfied by a designer experiment, in principle. The commensurability condition (41) is satisfied by the choice of neutrino energy. Reference to Fig. 1a reveals that for $E$ between the two resonant energies, a linear relation holds:

$$
E \approx \frac{\delta m_{\mp}^{2}}{\left.\delta m_{32}^{2}\right|_{\mathrm{v}}} E_{R}
$$

where here and in what follows the $\mp$ holds for $E>\frac{1}{2} E_{R}$. This equation leads to the desired constraint on the requisite energy:

$$
E=\frac{\Delta_{\mp}^{\mathrm{m}}}{\Delta_{-}^{\mathrm{m}}+\Delta_{+}^{\mathrm{m}}} E_{R}=\frac{n_{\mp}}{n_{-}+n_{+}} E_{R}
$$

The requisite length is obtained from $L=4 E \Delta_{ \pm} / \delta m_{ \pm}^{2}$. Substituting for $E$ from eq. (42) and for $\Delta_{\mp}^{\mathrm{m}}$ from eq. (39), one gets

$$
L=\frac{4 \pi}{3} \frac{E_{R}}{\left.\delta m_{32}^{2}\right|_{\mathrm{v}}} n_{\mp} .
$$

To summarize these results, the T-violating oscillation asymmetry is maximized when the neutrino energy satisfies eq. (43), and the length satisfies eq. (44). At fixed optimizing energy $E$, i.e. fixed $n_{+} / n_{-}$, eq. (44) predicts recurring peaks at distances related to the distance to the first peak

$$
L_{1}=\frac{4 \pi}{3} \frac{E_{R}}{\left.\delta m_{32}^{2}\right|_{\mathrm{v}}} \sim 3600 \cos 2 \theta_{31}\left[\frac{1.5 \mathrm{~g} \mathrm{~cm}^{-3}}{Y_{e} \rho}\right] \mathrm{km}
$$

by $L=L_{1} \times n_{\mp}$, with $n_{\mp}$ any positive integer allowed by eqs. (43) and (40) (which excludes multiples of three, at a minimum).

Plotted against distance at fixed $E$, each peak will exhibit the same height since the prefactor $J_{\mathrm{m}}$ depends on energy but not distance. One may ask what energy optimizes the peak heights. The prefactor $J_{\mathrm{m}}$ is maximized when $\frac{\delta m_{+}^{2}}{\delta m_{-}^{2}}$ is maximized. From eqs. (41) and (33), one finds

$$
\delta m_{\mp}^{2}=\left.\frac{n_{\mp}}{n_{-}+n_{+}} \delta m_{32}^{2}\right|_{\mathrm{v}},
$$

which, when inserted into eq. (『), yields

$$
\frac{J_{\mathrm{m}}}{J_{\mathrm{v}}}=\frac{\left(n_{+}+n_{-}\right)^{2}}{n_{+} n_{-}} \frac{\left.\delta m_{21}^{2}\right|_{\mathrm{v}}}{\left.\delta m_{32}^{2}\right|_{\mathrm{v}}}
$$


and so the heights of the asymmetry peaks are

$$
6 \sqrt{3} \frac{\left(n_{+}+n_{-}\right)^{2}}{n_{+} n_{-}} \frac{\left.\delta m_{21}^{2}\right|_{\mathrm{v}}}{\left.\delta m_{32}^{2}\right|_{\mathrm{v}}} .
$$

In terms of $E$, given in eq. (42), these asymmetry heights are

$$
6 \sqrt{3} \frac{E_{R}^{2}}{E\left(E_{R}-E\right)} \frac{\left.\delta m_{21}^{2}\right|_{\mathrm{v}}}{\left.\delta m_{32}^{2}\right|_{\mathrm{v}}} .
$$

As $E$ approaches the low-energy resonant value near zero or the high-energy resonant value $E_{R}$, the assumption that the $\delta m^{2}$ 's vary linearly with energy fails and our formulae here become invalid. 